\title{
CT Attenuation Analysis of Carotid Intraplaque Hemorrhage
}

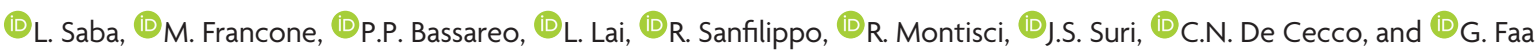

\section{ABSTRACT}

BACKGROUND AND PURPOSE: Intraplaque hemorrhage is considered a leading parameter of carotid plaque vulnerability. Our purpose was to assess the CT characteristics of intraplaque hemorrhage with histopathologic correlation to identify features that allow for confirming or ruling out the intraplaque hemorrhage.

MATERIALS AND METHODS: This retrospective study included 91 patients (67 men; median age, $65 \pm 7$ years; age range, 41-83 years) who underwent CT angiography and carotid endarterectomy from March 2010 to May 2013. Histopathologic analysis was performed for the tissue characterization and identification of intraplaque hemorrhage. Two observers assessed the plaque's attenuation values by using an $\mathrm{ROI}\left(\geq 1\right.$ and $\leq 2 \mathrm{~mm}^{2}$ ). Receiver operating characteristic curve, Mann-Whitney, and Wilcoxon analyses were performed.

RESULTS: A total of 169 slices were assessed (59 intraplaque hemorrhage, 63 lipid-rich necrotic core, and 47 fibrous); the average values of the intraplaque hemorrhage, lipid-rich necrotic core, and fibrous tissue were 17.475 Hounsfield units (HU) and 18.407 HU, $39.476 \mathrm{HU}$ and $48.048 \mathrm{HU}$, and $91.66 \mathrm{HU}$ and $93.128 \mathrm{HU}$, respectively, before and after the administration of contrast medium. The Mann-Whitney test showed a statistically significant difference of $\mathrm{HU}$ values both in basal and after the administration of contrast material phase. Receiver operating characteristic analysis showed a statistical association between intraplaque hemorrhage and low $\mathrm{HU}$ values, and a threshold of $25 \mathrm{HU}$ demonstrated the presence of intraplaque hemorrhage with a sensitivity and specificity of $93.22 \%$ and $92.73 \%$, respectively. The Wilcoxon test showed that the attenuation of the plaque before and after administration of contrast material is different (intraplaque hemorrhage, lipid-rich necrotic core, and fibrous tissue had $P$ values of $.006, .0001$, and .018 , respectively).

CONCLUSIONS: The results of this preliminary study suggest that CT can be used to identify the presence of intraplaque hemorrhage according to the attenuation. A threshold of $25 \mathrm{HU}$ in the volume acquired after the administration of contrast medium is associated with an optimal sensitivity and specificity. Special care should be given to the correct identification of the ROI.

ABBREVIATIONS: $\mathrm{Az}=$ area under the receiver operating characteristic curve; $\mathrm{CEA}=$ carotid endarterectomy; $\mathrm{HU}=$ Hounsfield unit; IPH $=$ intraplaque hemorrhage; LRNC $=$ lipid-rich necrotic core

D uring the past few years, several studies have demonstrated that the occurrence of intraplaque hemorrhage (IPH) in the carotid artery is linked with an increased risk of plaque rupture, ${ }^{1-3}$

Received April 10, 2017; accepted after revision August 20.

From the Departments of Radiology (L.S.), Cardiology (M.F., P.P.B., G.F.), and Vascular Surgery (R.S., R.M.), Azienda Ospedaliero Universitaria of Cagliari - Polo di Monserrato, Cagliari, Italy; Department of Radiological, Oncological, and Pathological Sciences (L.L.), Sapienza University of Rome, Rome, Italy; Point of Care Devices (J.S.S.), Global Biomedical Technologies, Roseville, California; AtheroPoint (I.S.S.), Roseville, California; Department of Electrical Engineering (I.S.S.), Idaho State University, Pocatello, Idaho; and Department of Radiology and Radiological Science (C.N.D.C.), Division of Cardiovascular Imaging, Medical University of South Carolina, Charleston, South Carolina.

Please address correspondence to Luca Saba, MD, Department of Radiology, Azienda Ospedaliero Universitaria (A.O.U.), di Cagliari - Polo di Monserrato s.s. 554 Monserrato (Cagliari) 09045, Italy; e-mail: lucasaba@tiscali.it

http://dx.doi.org/10.3174/ajnr.A5461 and therefore, IPH is considered a fundamental parameter of the so-called plaque vulnerability. ${ }^{4}$

Most of the research performed in the past 20 years was based on results from autopsy studies or on the specimens taken from carotid endartectomies. ${ }^{5,6}$ With the introduction of high-resolution black-blood MRI, ${ }^{7,8}$ it has become possible to identify noninvasively the IPH with optimal correlation to histology, and past literature has suggested an association between IPH and the occurrence of cerebrovascular events. ${ }^{9}$ In a recently published metaanalysis by Saam et al, ${ }^{10}$ it was demonstrated that the presence of IPH is associated with a 5- to 7-fold higher risk of cerebrovascular events.

However, carotid arteries are frequently imaged by using CT, which allows for characterizing the degree of stenosis ${ }^{11,12}$ with an excellent level of detail. Further, it offers information related to the risk of the plaque vulnerability such as the volume of the 
plaque and it subcomponents ${ }^{13,14}$ or the identification of ulcers in the plaque. ${ }^{15,16}$ Moreover, CT provides a unique option in some categories of patients who cannot be imaged by using MR imaging (eg, those with a prosthesis or pacemaker). Therefore, the identification of the determinants of plaque vulnerability, and in particular IPH, could be extremely important.

The purpose of this study was to assess the CT characteristics of the IPH with histopathologic correlation to identify features that allow for confirming or ruling out the IPH.

\section{MATERIALS AND METHODS Study Design and Patient Population}

The institutional review board of our hospital (Azienda Ospedaliera Universitaria di Cagliari) approved this retrospective study protocol, and we included all the patients who underwent carotid endarterectomy (CEA) and whose carotid specimen was analyzed by histologists. Therefore, 91 patients (67 men; median age, $65 \pm 7$ years; age range, $41-83$ years) who underwent CEA from March 2010 to May 2013 were included. This research was conducted in accordance with the guidelines of our institution's research committee, and because of the retrospective nature of the analysis, patient consent was waived.

We considered as an inclusion criterion that the CEA was performed within 10 days after the CTA, whereas we excluded those patients who underwent CEA for restenosis or radiation therapyinduced carotid stenosis. A further exclusion criterion was the plaque's rupture or fragmentation during the CEA procedure and/or manipulation.

In our hospital, the quantification of the degree of stenosis and the carotid artery plaque analysis is performed by using CTA according to previously published inclusion criteria. ${ }^{17,18}$ Carotid sonography is used as the first-line technique to assess the presence of atherosclerosis, and briefly, CTA of carotid arteries is performed when 1) carotid sonography shows a pathologic stenosis $\left(>50 \%\right.$ measured with the NASCET criteria ${ }^{19}$ ) or features related to plaque vulnerability (eg, ulcerations, irregular surface) and 2) sonography cannot adequately assess the degree of stenosis and the plaque's characteristics because of anatomic conditions. $\mathrm{Pa}-$ tients were also classified as symptomatic or asymptomatic according to the neurologic assessment documented in the clinical chart review by using the Trial of ORG 10172 in Acute Stroke Treatment (TOAST) criteria. Part of the study population $(n=$ 58) of this research was included in previously published papers. $^{20,21}$

\section{CTA Technique}

Patients were studied by using a 16-multidetector row CT system (Brilliance; Philips Healthcare, Best, the Netherlands). CT images were obtained with coverage from the aortic arch to the carotid siphon with caudocranial direction. Examinations were performed before and after the administration of contrast material. Angiographic phase was obtained with the administration of 80 $\mathrm{mL}$ of prewarmed contrast medium (Ultravist 370; Bayer HealthCare, Berlin, Germany). CT technical parameters included: slice thickness, $0.6 \mathrm{~mm}$; interval, $0.3 \mathrm{~mm}$; matrix size, $512 \times 512$; and field of view, 14-19 cm. A C-filter algorithm of reconstruction was applied.

\section{Carotid Endarterectomy}

Two vascular surgeons (R.M. and R.S., with 25 and 16 years of experience, respectively) performed all the CEA procedures by using the "en-bloc" technique by scoring the outer media and adventitia with a scalpel and then removing the plaque as an intact tube. This approach was adopted to reduce the manipulation of the carotid artery and avoid the potential rupture/fragmentation of the plaque.

In our hospital, patients underwent CEA according to the recommendations by the Asymptomatic Carotid Atherosclerosis Study (ACAS), ${ }^{22}$ NASCET, ${ }^{19}$ Stroke Prevention and Educational Awareness Diffusion (SPREAD), ${ }^{23}$ and European Carotid Surgery Trial $(\mathrm{ECST})^{3}$ after the exclusion of a cardioembolic source of embolism.

\section{Histologic Analysis of the Plaque}

The methods used for the plaque processing and histologic analysis have been described in a previously published paper. ${ }^{20}$ In summary, the histologic analysis was performed by 2 histologists (G.F. and L.L., with 22 and 5 years of experience, respectively) who were blinded to the radiologic findings. After the CEA, the excised "en-bloc" plaque is immediately fixed in formalin, and it is sent to the laboratory directly after the surgical procedure. Carotid plaques were decalcified and embedded in paraffin wax. None of the CEA specimens showed disruption of the luminal surface of the plaque. The portion of the specimen that showed the carotid plaque was divided transversely in sections having $3 \mathrm{~mm}$ intervals that were air-dried at $60^{\circ} \mathrm{C}$ for 45 minutes. After this phase, paraffin was removed by xylol, and the sections were hydrated. Finally, endogenous peroxidase activity was blocked by $2 \%$ hydrogen peroxide $\left(\mathrm{H}_{2} \mathrm{O}_{2}\right)$. The transverse sections were subjected to histologic examination to identify the plaque components. Because the main focus of the study was to assess the attenuation characteristics of IPH, special care was used in the identification of IPH. Finally, according to the histologic characteristics, the plaque of each slice was categorized into one of 4 groups: IPH, lipidrich necrotic core (LRNC), fibrous, and calcified.

\section{Co-Registration and Histology Matching}

The co-registration between CTA and histologic slices represents a key point of this paper, and special care was dedicated to avoid misregistration. After the selection of the CTA slice, the corresponding histologic section was identified by considering the following parameters: distance from the carotid bifurcation, lumen shape, and presence of prominent areas of calcification. In particular, the location of the bifurcation was identified to measure the length in the $\mathrm{z}$-axis correctly. Moreover, given the difference in thickness between the MDCTA image sections $(0.625 \mathrm{~mm})$ and the histologic cross sections $(5 \mu \mathrm{m})$, to further validate, $2-3$ histologic sections were compared with 1 cross-sectional MDCTA image on the basis of the relative distance from the bifurcation.

\section{IPH and Plaque Analysis Quantification}

Two radiologists (L.S. and M.F., with 11 and 8 years of experience in CTA, respectively) performed all measurements of Hounsfield units (HU) by using as window-level settings a width of 850 and 


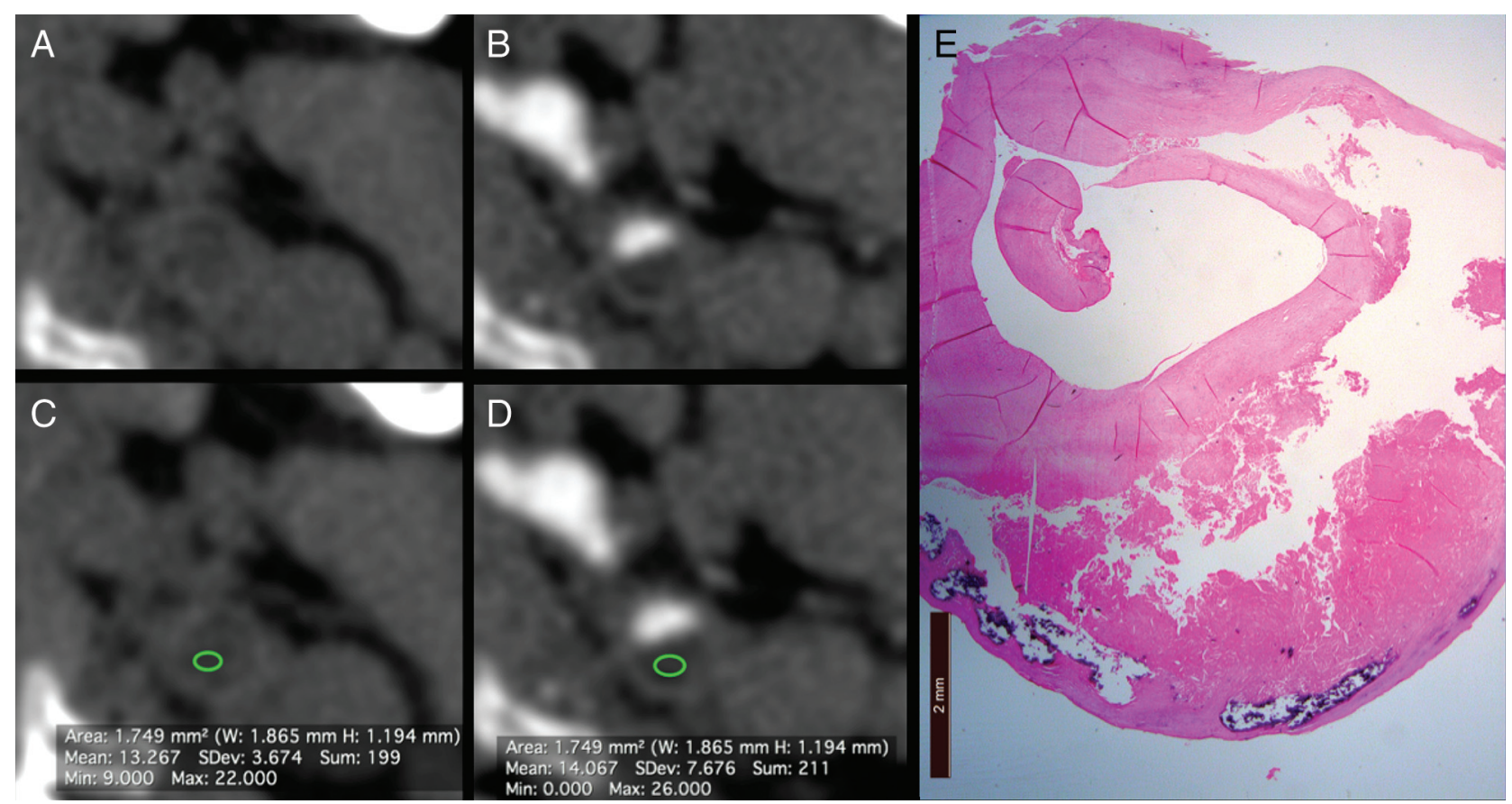

FIG 1. A 67-year-old male patient. The images acquired before $(A)$ and after the administration of contrast material (B) clearly show the carotid artery plaques. The ROI analyses before $(C)$ and after the administration of contrast material $(D)$ show the $\mathrm{HU}$ attenuation of the plaque (13.267 $\mathrm{HU}$ and $14.067 \mathrm{HU}$, respectively). In panel $E$, the corresponding histologic slice is given.

length of $300 .{ }^{24}$ In this study, the registration phase between CT and histologic slices was fundamental, and special care was used. The CT analysis was performed in 3 different phases. The first one was conducted with both radiologists in consensus to assess the image quality of the datasets and exclude those carotid arteries that showed dental streak artifacts or movement artifacts that reduced the image quality. The second and third phases were performed with radiologists who worked independently.

In the second phase, the angiographic dataset was assessed; radiologists had histopathologic sections as well as the spatial coordinates (slice number $-\mathrm{Z}$ distance from the carotid bifurcation), and they were asked to trace a circular or elliptical ROI ( $\geq$ $1 \mathrm{~mm}^{2}$ and $\leq 2 \mathrm{~mm}^{2}$ ) in the plaque and to quantify the HU attenuation values in the analyzed slice. The shape of the vessel wall and the lumen as well as the location of the bifurcation and the presence of calcifications in the plaque were used for matching. The procedures were repeated for each slice of each patient where it was possible to trace an ROI of at least $1 \mathrm{~mm}^{2}$.

In the third phase, radiologists selected the basal scan dataset and identified the same slice corresponding to the angiographic phase. To reach a correct registration between basal and angiographic datasets, they visually verified if the slices were registered or if it was necessary to select another slice along the z-axis. After this "matching phase," the same ROI used in the contrast phase (duplicate option) was positioned in the same position of the plaque to measure the attenuation values (Fig 1).

\section{Statistical Analysis}

In this study, the normality of each continuous variable group was tested by using the Kolmogorov-Smirnov $Z$ test, and appropriate tests for Gaussian or non-Gaussian values were selected. The Bland-Altman plot was calculated between the 2 readers to test the attenuation values, and the averaged value for each section was considered for the analysis. The Mann-Whitney test was calculated to test the difference of attenuation values between the different categories (IPH, LRNC, and fibrous tissue). Receiver operating characteristic curve analysis was performed, and the model was characterized by the area under the receiver operating characteristic curve (Az) with 95\% CIs to test the relationship between $\mathrm{HU}$ attenuation values in the ROI and IPH. Moreover, thresholds derived from the ROC analysis were calculated. The Wilcoxon test was applied to test the difference of attenuation values before and after administration of contrast material for the plaques (IPH, LRNC, and fibrous).

A $P$ value $<.05$ was regarded as indicating a statistically significant association, and all values were calculated by using a 2 -tailed significance level. $R$ statistical and computing software (www.rproject.org) was used for statistical analyses.

\section{RESULTS \\ General Results}

The mean time interval between CT and CEA was 9 days (range, 3-14 days). No patients were excluded from the analysis. Twentysix cases of IPH were found (prevalence of $23.08 \%$ ), and another 21 had LRNC without IPH (prevalence 23.07\%). In 5 cases, along the length of the plaque, there were histologic slices with LRNC and other slices with IPH (prevalence of 5.45\%). The other 39 plaques did not show IPH or LRNC and were mainly calcified $(n=24)$ or characterized by fibrous tissue or myofibroblast proliferation $(n=15)$.

In our cohort, we found 29 asymptomatic patients and 62 symptomatic patients. (In the 62 symptomatic patients, 25 strokes, 24 transient ischemic attacks, and 13 cases of amaurosis fugax were observed.) The analysis of the association between IPH 
Table 1: Summary of attenuation values

\begin{tabular}{lcccccc}
\hline Type of Tissue & $\mathbf{N}$ & Mean HU & SD & Median & $\begin{array}{c}\mathbf{2 . 5 - 9 7 . 5} \\
\text { Percentiles }\end{array}$ & $\begin{array}{c}\text { Normal } \\
\text { Distribution }\end{array}$ \\
\hline Basal & & & & & & \\
$\quad$ Fibrous & 47 & 91.66 & 17.7474 & 93 & $47.525-121.400$ & 0.0052 \\
$\quad$ LRNC & 63 & 39.476 & 19.4529 & 34 & $16.075-87.625$ & 0.0355 \\
$\quad$ IPH & 59 & 17.475 & 4.9283 & 17 & $8.975-26.025$ & 0.0668 \\
Contrast & & & & & & \\
$\quad$ Fibrous & 47 & 93.128 & 16.3609 & 94 & $49.875-120.775$ & 0.0003 \\
$\quad$ LRNC & 63 & 48.048 & 19.3811 & 43 & $21.000-85.925$ & 0.1446 \\
IPH & 59 & 18.407 & 4.8428 & 18 & $10.975-27.000$ & 0.0021 \\
\hline
\end{tabular}

between $-4 \mathrm{HU}$ and $+5 \mathrm{HU}$ for LRNC, and between $-6 \mathrm{HU}$ and +12 for fibrous tissue.

\section{Mann-Whitney Test}

By comparing the attenuation values among the 3 different analyzed categories (IPH, LRNC, and fibrous), we found that there was a statistically significant difference of $\mathrm{HU}$ values both before and after the administration of contrast ma-
Table 2: Mann-Whitney analysis

\begin{tabular}{lccc}
\hline Type of Tissue & IPH & LRNC & Fibrous \\
\hline Basal & & & \\
IPH & $\mathrm{x}$ & 0.001 & 0.001 \\
LRNC & 0.001 & $\mathrm{x}$ & 0.001 \\
Fibrous & 0.001 & 0.001 & $\mathrm{x}$ \\
Contrast & & & \\
IPH & $\mathrm{x}$ & 0.001 & 0.001 \\
LRNC & 0.001 & $\mathrm{x}$ & 0.001 \\
Fibrous & 0.001 & 0.001 & $\mathrm{x}$ \\
\hline
\end{tabular}

Note:- $\mathrm{x}$ indicates test is not applicable.

and the presence of cerebrovascular symptoms (including the 5 plaques where some histologic slices had LRNC and others had IPH for a total of 31 subjects with IPH) revealed an association between plaques with IPH and symptoms with a $P$ value of .02 (17\% of IPH in asymptomatic subjects [5/29] and $42 \%$ of IPH in symptomatic subjects [26/62]).

A total of 435 histologic slices were recorded and measured (the median number for each plaque was 5, ranging from 3-12). We excluded 61 histologic sections because of suboptimal quality. Another 129 histologic sections were excluded because they were mainly calcified plaques, and in another 63 cases, a small amount of calcium was found in the histopathologic examinations. Therefore, 253 histologic sections were excluded.

Radiologists performed the CT matching in the remaining 182 histologic slices. They excluded 2 patients (with a total of 7 histologic slices) because of streak artifacts and 1 patient who had movement artifacts ( 6 histologic slices). The final number of assessed slices was 169 (59 IPH, 63 LRNC, and 47 fibrous).

\section{Plaque Analysis}

Table 1 summarizes the results of the attenuation values according to the histologic types in the arterial and venous phases. The average values of the IPH were 17.475 HU and 18.407 HU before and after the administration of contrast material, respectively. The average values of the LRNC were $39.476 \mathrm{HU}$ and $48.048 \mathrm{HU}$ before and after the administration of contrast material, respectively, whereas for the fibrous plaque, the average values were 91.66 HU and 93.128 HU before and after the administration of contrast material.

We found that the category that showed the bigger enhancement was the LRNC (average increase of $8.54 \mathrm{HU}$ ) followed by fibrous tissue (1.468 HU), whereas the IPH showed the smaller variation (average increase, 0.932 ). The concordance between the readers (measured by Bland-Altman plots) was good, with a systematic error (1.96 SD) between $-2 \mathrm{HU}$ and $+3 \mathrm{HU}$ for IPH, terial (Table 2). In Fig 2 a boxplot shows the attenuation values according to the category.

\section{Receiver Operating Characteristic Curve Analysis}

The receiver operating characteristic curve analysis was performed to test the association between the HU attenuation and the presence or absence of IPH, shown in Fig 3. The Az for the attenuation analysis performed in the basal scan was 0.944 , whereas the $\mathrm{Az}$ after the administration of contrast medium was 0.978 (difference between the Az; $P=.026$ ). From the receiver operating characteristic curve analysis, we also tested multiple thresholds to identify the best combination of sensitivity and specificity value (Table 3 ). We found that a threshold of $25 \mathrm{HU}$ in the plaque (in the dataset after administration of contrast material) has a sensitivity of $93.22 \%$ and a specificity of $92.73 \%$ for the presence of IPH.

\section{Wilcoxon Analysis}

This test was used to check the effect of the contrast material in the plaque categories. By comparing the overall attenuation of the plaques (IPH, LRNC, and fibrous) before and after the administration of contrast medium, we found a statistically significant difference $(P=.001)$. By assessing the attenuation of IPH, LRNC, and fibrous tissue before and after contrast medium, we found $P$ values of $.006, .0001$, and .018 , respectively, demonstrating that the attenuation of the plaque before and after administration of contrast material is different.

\section{DISCUSSION}

Recent published studies have demonstrated that the presence of IPH is related to an increased risk of stroke independent of the severity of luminal stenosis. ${ }^{25,26}$ Therefore, the identification of IPH represents an important step to correctly stratify the risk of potential cerebrovascular events of a subject.

CT is a widely used technique for the preoperative assessment of the subject, which allows for obtaining information related to other features related to the plaque's vulnerability such as the presence of ulcerations or large plaque volume. ${ }^{13,27}$ It is important to remember that because of the radiation delivered, CT is not considered the best tool for follow-up of patients with carotid artery stenosis (unlike ultrasound), and that is one of its main limitations as a diagnostic tool; however, the improvement in CT technology with the introduction of dose-reduction techniques results in a viable option. The purpose of this study was to assess the CT characteristics of IPH with histopathologic correlation to identify features that allow for confirming or ruling out the IPH. 


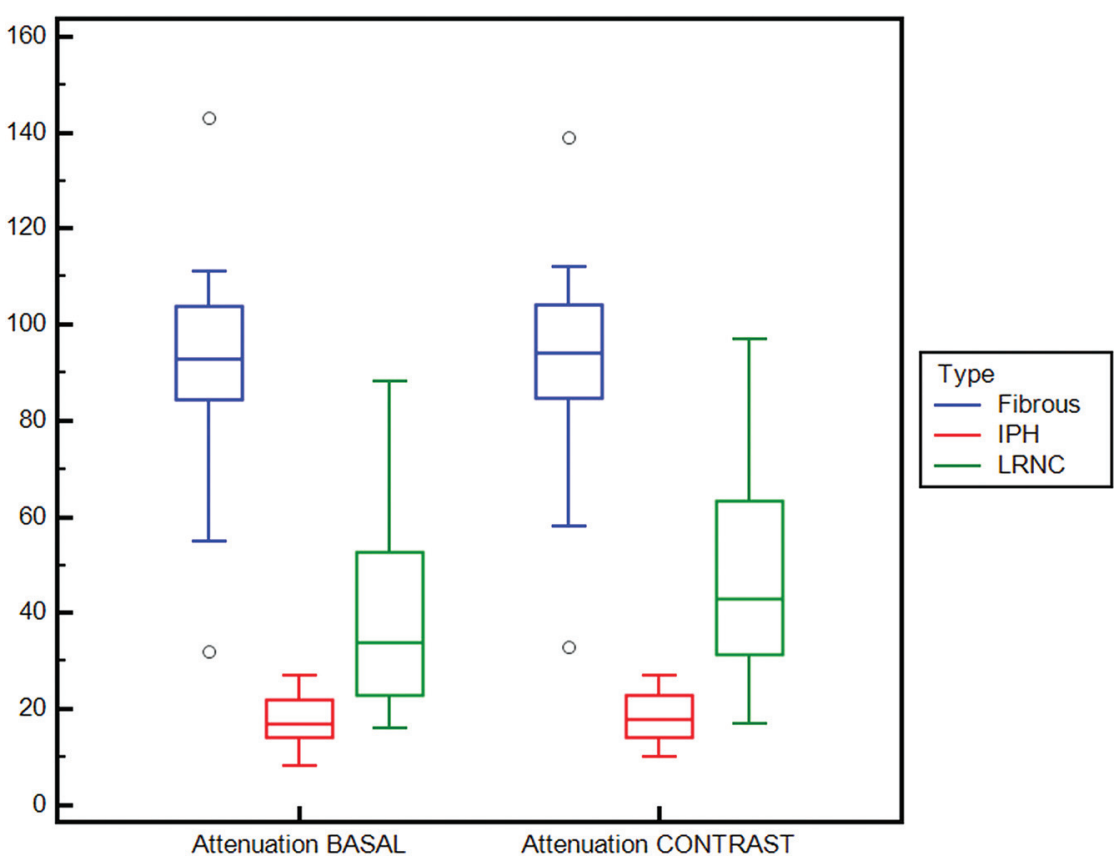

FIG 2. Boxplot with the attenuation values before and after the administration of contrast material according to the type of tissue.

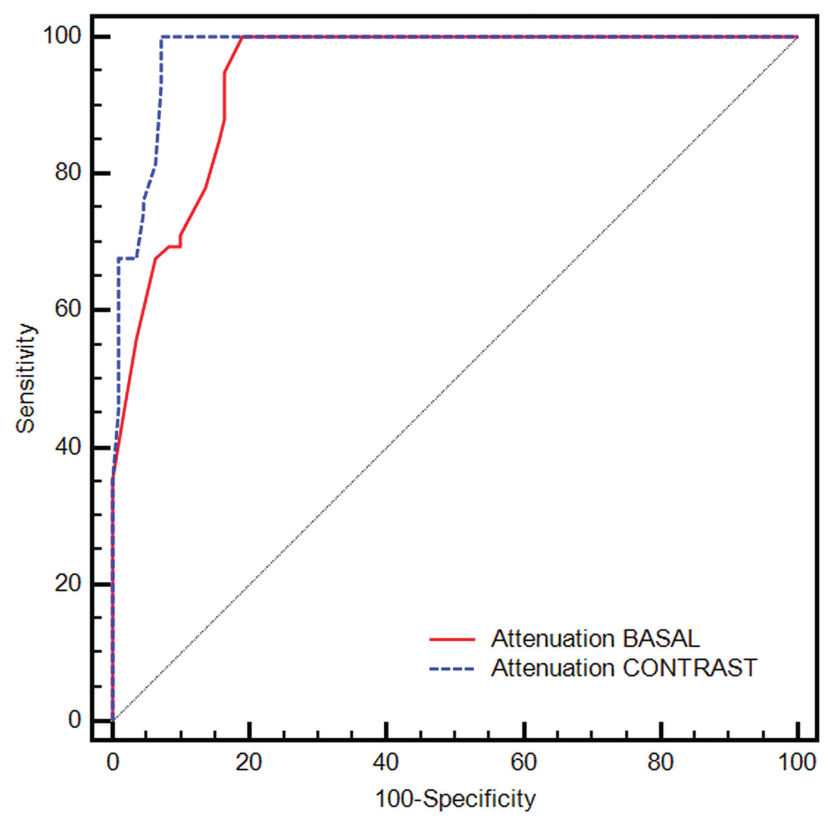

FIG 3. Receiver operating characteristic curve analysis that shows the Az of the attenuation (basal and after the administration of contrast medium) versus the presence/absence of IPH.

To obtain correct data, our readers (histologists and radiologists) gave special attention to excluding all those slices or CT datasets that were not optimal from a technical point of view. Moreover, the calcified plaques or those plaques with a visible cluster of calcium were excluded. First, the identification of the calcium is extremely easy thanks to the big attenuation that this tissue produces in the $\mathrm{x}$-rays. For the same reason, those histologic slices where calcium was visible in small amounts were excluded. From the original 435 histologic slices, radiologists analyzed 169 slices (38.9\%).
We found that the average value of the IPH was extremely low, with an average basal value of $17.475 \mathrm{HU}$ and average value of $18.407 \mathrm{HU}$ after the administration of contrast medium. In 2009, Adjuk et $\mathrm{al}^{28}$ found that the attenuation values of the American Heart Association plaque VIb (confluent extracellular lipid core complicated by hemorrhage) was lower compared with the other types of plaque, with an average value of $22 \mathrm{HU}$ compared with 59 HU for the other types of plaques, and these data were confirmed in a further publication performed by the same group in 2013. ${ }^{29}$ Our values are lower compared with those found by Adjuk et $\mathrm{al}^{28,29}$ and we think that this can be attributed to 2 different causes. First, the different selection criteria; in fact, we excluded all those plaques that also had a small cluster of calcium that can justify the raised attenuation values in the work of Adjuk et al. ${ }^{28,29}$ Second, we did not considered histologic slices with LRNC and IPH, but only those 2 features independently; this approach may explain the lower attenuation values of our observations. In addition, a study published by de Weert et $\mathrm{al}^{30}$ in 2006 demonstrated that the presence of very hypoattenuated areas was associated with the presence of plaques complicated by hemorrhage $(<30 \mathrm{HU})$.

We also compared the attenuation values among the 3 different analyzed categories (IPH, LRNC, and fibrous), and we found that there was a statistically significant difference of $\mathrm{HU}$ values (Table 2). This result demonstrates that the attenuation values are different from the attenuation of LRNC and fibrous tissue. In particular, hemorrhage has significantly lower attenuation values compared with the other categories of tissues.

The receiver operating characteristic curve analysis confirmed the strength of the association between the hypoattenuated values and presence of IPH ( $\mathrm{Az}=0.944$ in basal analysis and 0.978 after contrast medium), and by considering a threshold value of $25 \mathrm{HU}$ (in the scans obtained after the administration of contrast material), we obtained a sensitivity of $93.22 \%$ and a specificity of $92.73 \%$ for the presence of IPH. These results demonstrated that it is possible to identify an attenuation threshold by identifying the presence of IPH.

Our results are different from those observed by U-King-Im et $\mathrm{al}^{31}$ where the authors compared CTA and IPH detected by MR imaging and found that mean CT plaque attenuation was higher for plaques with MR imaging-defined IPH (47 HU) compared with plaques without IPH (43 HU). Moreover, they found a significant overlap between the distributions of plaque densities by concluding that mean plaque attenuation is of limited value in distinguishing between the 2 groups. The differences between our results and the paper by U-King-Im et $\mathrm{al}^{31}$ can be explained by the different methodology applied. They used as reference standard MR-defined IPH, whereas we considered the histopathologic 


\begin{tabular}{|c|c|c|c|c|c|c|c|c|}
\hline \multirow[b]{2}{*}{ HU Value } & \multicolumn{4}{|c|}{ Basal Values } & \multicolumn{4}{|c|}{ After Contrast Medium Values } \\
\hline & Sensitivity & Specificity & $+\mathrm{LR}$ & $-\mathrm{LR}$ & Sensitivity & Specificity & $+\mathrm{LR}$ & -LR \\
\hline$<0$ & 0.00 & 100.00 & NC & 1.00 & 0.00 & 100.00 & NC & 1.00 \\
\hline$\leq 2$ & 55.93 & 96.36 & 15.38 & 0.46 & 35.59 & 100.00 & NC & 0.64 \\
\hline$\leq 4$ & 67.80 & 93.64 & 10.65 & 0.34 & 45.76 & 99.09 & 50.34 & 0.55 \\
\hline$\leq 8$ & 69.49 & 90.00 & 6.95 & 0.34 & 67.80 & 99.09 & 74.58 & 0.32 \\
\hline$\leq 12$ & 71.19 & 90.00 & 7.12 & 0.32 & 67.80 & 96.36 & 18.64 & 0.33 \\
\hline$\leq 16$ & 77.97 & 86.36 & 5.72 & 0.26 & 74.58 & 95.45 & 16.41 & 0.27 \\
\hline$\leq 20$ & 84.75 & 84.55 & 5.48 & 0.18 & 76.27 & 95.45 & 16.78 & 0.25 \\
\hline$\leq 22$ & 88.14 & 83.64 & 5.39 & 0.14 & 81.36 & 93.64 & 12.78 & 0.20 \\
\hline$\leq 25$ & 94.92 & 83.64 & 5.80 & 0.061 & 93.22 & 92.73 & 12.82 & 0.073 \\
\hline$\leq 30$ & 100.00 & 80.91 & 5.24 & 0.00 & 100.00 & 92.73 & 13.75 & 0.00 \\
\hline$\leq 120$ & 100.00 & 0.00 & 1.00 & NC & 100.00 & 0.00 & 1.00 & NC \\
\hline
\end{tabular}

Note:-_LR indicates positive likelihood ratio; - LR, negative likelihood ratio; NC, not classifiable.

slices. We excluded from the analysis all the slices where a cluster of calcium was identified, and this explains the average lower CT values found in our study in those plaques with IPH. Moreover, we considered multiple sections for each plaque according to the histopathologic category, and this is important because we found 10 plaques where there were areas with IPH and others without IPH. These findings confirm that in the same plaques, there exist different tissues, and they are not always represented in a single axial section. For this reason, we suggest readers check the entire length of the plaque and not only the thickened point and assess with ROIs having different levels of the plaque. This is a significant point because the plaques show a spatial heterogeneity other than a temporal variability; therefore, the plaque should be considered a dynamic process, and lack of IPH doesn't mean that sometime in the future IPH cannot appear.

We also tested the difference in attenuation before and after the administration of contrast medium and found that there was a statistically significant difference $(.006, .0001$, and .018 for IPH, LRNC, and fibrous tissue, respectively), by confirming the fact that the contrast material plays an important role in the attenuation values of the carotid artery plaques. ${ }^{32-34}$

In this study, there are some limitations. First, this is a retrospective study, and this approach may determine a bias; however, it is our opinion this can be a considered a minor limitation because our focus was to assess the attenuation value of the IPH, and we would use the same paradigm in prospective approach. Second, the registration between histologic sections and the CT dataset is fundamental to obtain correct as well as relevant data. We tried to use our experience and all the potential tools to overcome the problems related to the registration (spatial registration features, manual and experienced correction, and the use of 2 different readers). However, there are some limits that are not possible to solve, in particular, the fact that the histologic preparation leads to plaque shrinkage ${ }^{35}$; in the study by de Weert et al, ${ }^{30}$ the total plaque area acquired in MDCTA images was 30\% larger than the histologic total plaque area.

\section{CONCLUSIONS}

The results of this preliminary study suggest that CT can be used to identify the presence of IPH according to the attenuation. A threshold of $25 \mathrm{HU}$ in the volume acquired after the administration of contrast medium is associated with an optimal sensitiv- ity and specificity. Special care should be given to the correct identification of the area to place the ROI.

Disclosures: Marco Francone_UNRELATED: Payment for Lectures Including Service On Speakers Bureaus: Bracco Medical Imaging. Carlo Nicola De Cecco-UNRELATED: Grants/Grants Pending: Siemens* . Money paid to the institution.

\section{REFERENCES}

1. Kwee RM, van Oostenbrugge RJ, Mess WH, et al. MRI of carotid atherosclerosis to identify TIA and stroke patients who are at risk of a recurrence. J Magn Reson Imaging 2013;37:1189-94 CrossRef Medline

2. Mono ML, Karameshev A, Slotboom J, et al. Plaque characteristics of asymptomatic carotid stenosis and risk of stroke. Cerebrovasc Dis 2012;34:343-50 CrossRef Medline

3. Kurosaki Y, Yoshida K, Endo H, et al. Association between carotid atherosclerosis plaque with high signal intensity on T1-weighted imaging and subsequent ipsilateral ischemic events. Neurosurgery 2011;68:62-67; discussion 67 CrossRef Medline

4. Demarco JK, Huston J 3rd. Imaging of high-risk carotid artery plaques: current status and future directions. Neurosurg Focus 2014; 36:E1 CrossRef Medline

5. Peeters W, Hellings WE, de Kleijn DP, et al. Carotid atherosclerotic plaques stabilize after stroke: insights into the natural process of atherosclerotic plaque stabilization. Arterioscler Thromb Vasc Biol 2009;29:128-33 CrossRef Medline

6. Hellings WE, Peeters W, Moll FL, et al. Composition of carotid atherosclerotic plaque is associated with cardiovascular outcome: a prognostic study. Circulation 2010;121:1941-50 CrossRef Medline

7. Moody AR, Allder S, Lennox G, et al. Direct magnetic resonance imaging of carotid artery thrombus in acute stroke. Lancet 1999; 353:122-23 CrossRef Medline

8. Daniels LR, Gladman JR, Altaf N, et al. Magnetic resonance direct thrombus imaging in moderate carotid artery stenosis. Stroke 2006; 37:767-68 CrossRef Medline

9. Millon A, Mathevet JL, Boussel L, et al. High-resolution magnetic resonance imaging of carotid atherosclerosis identifies vulnerable carotid plaques. J Vasc Surg 2013;57:1046-51.e2 CrossRef Medline

10. Saam T, Hetterich H, Hoffmann V, et al. Meta-analysis and systematic review of the predictive value of carotid plaque hemorrhage on cerebrovascular events by magnetic resonance imaging. J Am Coll Cardiol 2013;62:1081-91 CrossRef Medline

11. Anzidei M, Napoli A, Zaccagna F, et al. Diagnostic accuracy of colour Doppler ultrasonography, CT angiography and blood-pool-enhanced MR angiography in assessing carotid stenosis: a comparative study with DSA in 170 patients. Radiol Med 2012;117:54-71 CrossRef Medline

12. Lell M, Fellner C, Baum U, et al. Evaluation of carotid artery stenosis with multisection CT and MR imaging: influence of imaging mo- 
dality and postprocessing. AJNR Am J Neuroradiol 2007;28:104-10 Medline

13. van den Bouwhuijsen QJ, Bos D, Ikram MA, et al. Coexistence of calcification, intraplaque hemorrhage and lipid core within the asymptomatic atherosclerotic carotid plaque: The Rotterdam Study. Cerebrovasc Dis 2015;39:319-24 CrossRef Medline

14. Saba L, Raz E, Anzidei M, et al. Differences in plaque morphology and correlation of stenosis at the carotid artery bifurcation and the carotid siphon. AJR Am J Roentgenol 2013;201:1108-14 CrossRef Medline

15. Homburg PJ, Rozie S, van Gils MJ, et al. Atherosclerotic plaque ulceration in the symptomatic internal carotid artery is associated with nonlacunar ischemic stroke. Stroke 2010;41:1151-56 CrossRef Medline

16. Saba L, Caddeo G, Sanfilippo R, et al. CT and ultrasound in the study of ulcerated carotid plaque compared with surgical results: potentialities and advantages of multidetector row CT angiography. AJNR Am J Neuroradiol 2007;28:1061-66 CrossRef Medline

17. Saba L, Sanfilippo R, Montisci R, et al. Agreement between multidetector-row CT angiography and ultrasound echo-color Doppler in the evaluation of carotid artery stenosis. Cerebrovasc Dis 2008;26: 525-32 CrossRef Medline

18. Saba L, Sanfilippo R, Pascalis L, et al. Carotid artery wall thickness and ischemic symptoms: evaluation using multi-detector-row CT angiography. Eur Radiol 2008;18:1962-71 CrossRef Medline

19. North American Symptomatic Carotid Endarterectomy Trial Collaborators. Beneficial effect of carotid endarterectomy in symptomatic patients with high-grade carotid stenosis. N Engl J Med 1991;325: 445-53 CrossRef Medline

20. Saba L, Tamponi E, Raz E, et al. Correlation between fissured fibrous cap and contrast enhancement: preliminary results with the use of CTA and histologic validation. AJNR Am J Neuroradiol 2014; 35:754-59 CrossRef Medline

21. Saba L, Raz E, Grassi R, et al. Association between the volume of carotid artery plaque and its subcomponents and the volume of white matter lesions in patients selected for endarterectomy. $A J R$ Am J Roentgenol 2013;201:W747-52 CrossRef Medline

22. Executive Committee for the Asymptomatic Carotid Atherosclerosis Study. Endarterectomy for asymptomatic carotid artery stenosis. JAMA 1995;273:1421-28 CrossRef Medline

23. Setacci C, Lanza G, Ricci S, et al. SPREAD Italian guidelines for stroke. Indications for carotid endarterectomy and stenting. J Cardiovasc Surg (Torino) 2009;50:171-82 Medline

24. Saba L, Mallarin G. Window settings for the study of calcified ca- rotid plaques with multidetector CT angiography. AJNR Am J Neuroradiol 2009;30:1445-50 CrossRef Medline

25. Parmar JP, Rogers WJ, Mugler JP 3rd, et al. Magnetic resonance imaging of carotid atherosclerotic plaque in clinically suspected acute transient ischemic attack and acute ischemic stroke. Circulation 2010;122:2031-38 CrossRef Medline

26. Takaya N, Yuan C, Chu B, et al. Presence of intraplaque hemorrhage stimulates progression of carotid atherosclerotic plaques: a highresolution magnetic resonance imaging study. Circulation 2005; 111:2768-75 CrossRef Medline

27. Rozie S, de Weert TT, de Monyé C, et al. Atherosclerotic plaque volume and composition in symptomatic carotid arteries assessed with multidetector CT angiography; relationship with severity of stenosis and cardiovascular risk factors. Eur Radiol 2009;19:2294301 CrossRef Medline

28. Ajduk M, Pavić L, Bulimbasić S, et al. Multidetector-row computed tomography in evaluation of atherosclerotic carotid plaques complicated with intraplaque hemorrhage. Ann Vasc Surg 2009;23: 186-93 CrossRef Medline

29. Ajduk M, Bulimbasić S, Pavić L, et al. Comparison of multidetectorrow computed tomography and duplex Doppler ultrasonography in detecting atherosclerotic carotid plaques complicated with intraplaque hemorrhage. Coll Antropol 2013;37:213-29 Medline

30. de Weert TT, Ouhlous $M$, Meijering E, et al. In vivo characterization and quantification of atherosclerotic carotid plaque components with multidetector computed tomography and histopathological correlation. Arterioscler Thromb Vasc Biol 2006;26:2366-72 CrossRef Medline

31. U-King-Im JM, Fox AJ, Aviv RI, et al. Characterization of carotid plaque hemorrhage: a CT angiography and MR intraplaque hemorrhage study. Stroke 2010;41:1623-29 CrossRef Medline

32. Saba L, Lai ML, Montisci R, et al. Association between carotid plaque enhancement shown by multidetector CT angiography and histologically validated microvessel density. Eur Radiol 2012;22:2237-45 CrossRef Medline

33. Saba L, Piga M, Raz E, et al. Carotid artery plaque classification: does contrast enhancement play a significant role? AJNR Am J Neuroradiol 2012;33:1814-17 CrossRef Medline

34. Saba L, Mallarini G. Carotid plaque enhancement and symptom correlations: an evaluation by using multidetector row $\mathrm{CT}$ angiography. AJNR Am J Neuroradiol 2011;32:1919-25 CrossRef Medline

35. Dobrin PB. Effect of histologic preparation on the cross-sectional area of arterial rings. J Surg Res 1996;61:413-15 CrossRef Medline 